\title{
Nonlinear Signal Processing
}

\author{
Martin Centurion, Mankei Tsang, Demetri Psaltis \\ California Institute of Technology, Pasadena, CA
}

We have studied the propagation of femtosecond pulses in a Kerr medium both experimentally and numerically. The nonlinear propagation in a Kerr material leads to the formation of optical patterns and filaments [1]. We investigate the similarities between the propagation of light pulses in a Kerr medium and the evolution of fluid dynamical systems, and propose the use of an optical system to simulate fluid dynamics.

The experiments were performed using a TiSapphire amplified laser system which generates 150femtosecond pulses with energies up to $2 \mathrm{~mJ}$ at a wavelength of $800 \mathrm{~nm}$. Carbon disulfide was chosen as the nonlinear medium due to its large nonlinear coefficient. A collimated beam with a diameter of $5 \mathrm{~mm}$ was launched into the nonlinear medium and the output beam profile was imaged on a CCD camera for different propagation lengths. Figure 1a shows an image of the central part $(0.5 \mathrm{~mm} \times 0.5 \mathrm{~mm})$ of the input beam and Fig. $1 \mathrm{~b}$ shows the output beam after traversing $10 \mathrm{~mm}$ of the nonlinear medium. Modulation instability causes the beam to break up into a pattern of connected lines (constellation) and bright spots (filaments). The output beam profile is repeatable over multiple laser shots provided the input beam does not change. The constellation is experimentally observed to appear before the filaments, and remains constant as the beam propagates [2]. After the constellation appears, the lines start to break up into an increasing number of filaments as the beam propagates [2,3]. The filaments reach a stable diameter of 12 micrometers and propagate for several millimeters before diverging. Increasing the energy of the input beam causes the beam to break up in a shorter distance and the formation of more filaments. The size and intensity of the filaments, however, does not change. We have also observed conical emission from the filaments which interacts with the constellation to seed the formation of new filaments.

Input beam

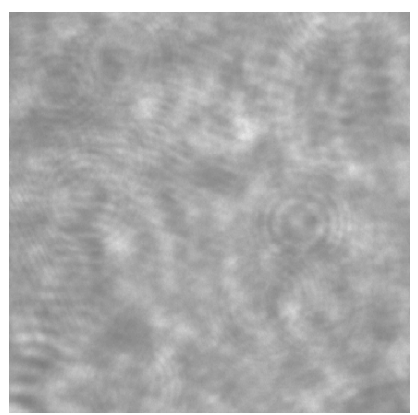

(a)
Output

(Experimental)

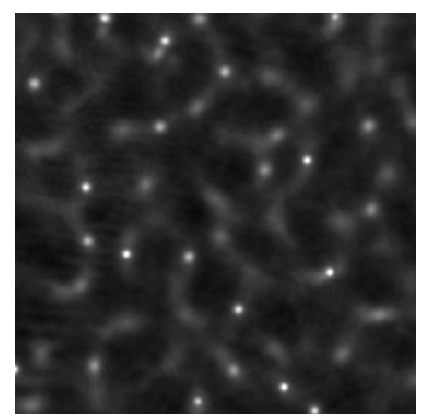

(b)

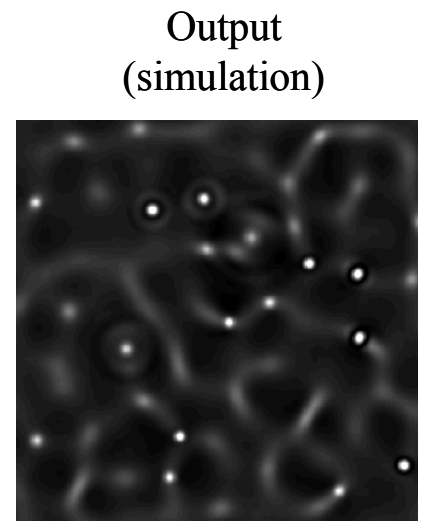

(c)

Figure 1. The amplitude of the input beam (a) was measured and used as the input of the numerical simulation. There is good agreement between the experimental (b) and simulated beam profile (c). The images are $0.5 \mathrm{~mm} \times 0.5 \mathrm{~mm}$.

The light propagation was also simulated numerically. Figure 1c shows the output beam calculated numerically. The propagation was simulated using a time-averaged nonlinear Schrodinger equation (NLSE), including the effects of diffraction, a positive third-order nonlinearity $\left(n_{2}\right)$ and a negative fifthorder nonlinearity $\left(n_{4}\right)$. The third-order nonlinearity generates an index change proportional to the intensity that causes self-focusing. The fifth-order term is responsible for the formation of stable filaments as it 
provides a mechanism to saturate the index change [4]. The intensity of the filaments measured experimentally is well below the threshold for plasma formation. The image of the input beam was used as the input intensity for the simulation, while the phase of the beam was assumed to be uniform. This provides a good approximation to spatial noise in the input beam, although an exact match cannot be expected since the phase information is missing. There is good qualitative agreement between the experimental and numerical output beams. The simulation shows the formation of the constellation and stable filaments, and the conical emission from the filaments.

In the case of light propagation through a medium with a negative $n_{2}$ (defocusing nonlinearity), it is possible to generate stable dark solitons which have a spiraling phase profile. These solitons carry angular momentum, and are also called optical vortex solitons, due to the rotating phase. Stable optical vortices have been observed experimentally in a defocusing Kerr medium [5]. It has also been predicted that stable optical vortices can be generated in materials with a cubic-quintic nonlinearity, where there is a positive $n_{2}$ and a negative $n_{4}$ [6]. Optical vortices are analogous to the discrete optical vortices observed in superfluids $[6,7]$, which is not surprising since the NLSE can be used to describe both the propagation of optical pulses and the dynamics of superfluids. In addition, it has been shown that the NLSE can be transformed to hydrodynamical equations using the Madelung transformation [8] and that the NLSE exhibits classical turbulence behavior [9]. The similarities between the mathematical description of optical and fluidic systems suggests that one could be used to compute the evolution of the other, provided the right parameters can be found.

We have investigated the use an optical system to compute the evolution of fluidic systems. The advantages of using an optical system are the inherent high speed, parallelism and configurability of optics. For a direct correspondence between optics and classical hydrodynamics it is essential to relate the quantized vortex lines to the distributed vorticity. The continuous vorticity can be approximated by spatially averaging the discrete vortices in the optical system. In an ordinary fluid, the creation of vortices is mainly due to an obstacle in the flow, the boundary of which in most cases is "no-slip". The vorticity naturally generated at such a boundary is diffused into the main flow by viscosity. In optics one can only have a "free-slip" boundary as enforced by the refractive index. In a completely incompressible fluid, a "free-slip" boundary only results in a potential flow and does not generate vorticity. We have found that it is possible to generate vorticity in an optical system by appropriate choice of the optical boundary conditions.

\section{References}

[1]. Y. Kivshar, D. Pelinovsky, "Self-focusing and transverse instabilities of solitary waves," Phys. Rep. 331, 118 (2000).

[2]. M. Centurion, Y. Pu, M. Tsang, D. Psaltis, "Dynamics of filament formation in a Kerr medium," Phys. Rev. A 71063811 (2005).

[3]. M. Centurion, Y. Pu, D. Psaltis, "Self-organization of spatial solitons," Opt. Express 13, 6202 (2005).

[4]. A. Piekara, "On self-trapping of a laser beam," IEEE J. Quantum Electron. QE-2, 249 (1966).

[5]. G. A. Swartzlander, Jr. and C. T. law, "Optical Vortex Solitons Observed in Kerr Nonlinear Media," Phys. Rev. Lett. 69, 2503 (1992).

[6]. M. J. Paz-Alonso, H. Michinel, "Superfluidlike Motion of Vortices in Light Condensates," Phys. Rev. Lett. 94, 093901 (2005).

[7]. Y. Pomeau and S. Rica, "Nonlinear diffraction," C.R. Acad. Sci. Paris 317, 1287 (1993).

[8]. E. Madelung, "Quantetheorie in hydrodynamischer form," Z. Phys. 40322 (1927).

[9] M. Abid, C. Huepe, S. Metens, C. Nore, C. T. Pham, L. S. Tuckerman, M. E. Brachet, "Gross-Pitaevskii dynamics of Bose-Einstein condensates and superfluid turbulence," Fluid Dynamics Research 33, 509 (2003), and references therein. 